\title{
A rare case of primary hypogonadism \& partial hypopituitarism in Klinefelter Syndrome.
}

\section{Shoib Ur Rehman, Rupa Ahluwalia}

\section{Background}

- Klinefelter syndrome is the most common genetic cause of primary hypogonadism in men .(1)

- It can present with a wide spectrum of phenotypical and hormonal abnormalities but only $10 \%$ of the patients get diagnosed before puberty

- Karyotype is $47 X X Y$ in $~ 80 \%$ of the cases and $20 \%$ have variable mosaic chromosomal aneuploidies $(2,3)$.

- Concomitant pituitary disease is uncommon in this chromosomal aneuploidy syndrome.

- We describe a rare case of Klinfelter syndrome with growth hormone and adrenocortical deficiency

\section{Case History}

We report a case of a thirty six year old man referred to the endocrine clinic with infertility and azoospermia on semen analysis. Relevant past medical history included nephrolithiasis, hiatus hernia and tinnitus. There was no history of previous use of exogenous glucocorticoids or anabolic steroids.

Examination revealed normal secondary sexual characteristics(tanner stage 5) but with evidence of bilateral microorchidia(USG confirmed testicular volumes around 2 cc)

Basal hormone levels were suggestive of hypergonadotrophic eugonadal state(Table 1). Given a low baseline cortisol (108nmol/L), dynamic tests were performed to further evaluation of the hypothalamic-pituitary-adrenal axis. Diagnosis of Klinefelter syndrome was made based on karyotyping.

MRI Pituitary was normal and DEXA scan revealed mild osteopenia in Lumbar spine (T-Score -1.2).

\begin{tabular}{|c|c|c|}
\hline Table 1 & Value & Range \\
\hline FSH & $37.7 \mathrm{IU} / \mathrm{L}$ & 1-12 IU/L \\
\hline LH & $21.4 \mathrm{IU} / \mathrm{L}$ & 0.6-12 IU/L \\
\hline Testosterone & $14.2 \mathrm{nmol} / \mathrm{L}$ & $9.9-27.8 \mathrm{nmol} / \mathrm{L}$ \\
\hline TSH & $1.41 \mathrm{mU} / \mathrm{L}$ & $0.35-3.5 \mathrm{mU} / \mathrm{L}$ \\
\hline FT4 & $10 \mathrm{pmol} / \mathrm{L}$ & 8-21 pmol/L \\
\hline IGF1 & $17.2 \mathrm{nmol} / \mathrm{L}$ & $9.6-32.8 \mathrm{nmol} / \mathrm{L}$ \\
\hline $\begin{array}{c}\text { SST Cortisol } \\
0(\min )\end{array}$ & $175 \mathrm{nmol} / \mathrm{L}$ & \\
\hline $\begin{array}{l}\text { Cortisol } \\
30 \text { (min) }\end{array}$ & $429 \mathrm{nmol} / \mathrm{L}$ & $>450 \mathrm{nmol} / \mathrm{L}$ \\
\hline $\begin{array}{l}\text { Cortisol } \\
60(\min )\end{array}$ & $487 \mathrm{nmol} / \mathrm{L}$ & \\
\hline
\end{tabular}

Insulin Tolerance Test

\begin{tabular}{|l|l|l|l|}
\hline $\begin{array}{l}\text { Time, } \\
\mathbf{m i n}\end{array}$ & $\begin{array}{l}\text { Glucose, } \\
\mathbf{m m o l} / \mathbf{L}\end{array}$ & $\begin{array}{l}\text { Cortisol, } \\
\text { nmol/L }\end{array}$ & $\begin{array}{l}\text { Growth } \\
\text { Hormone, } \\
\text { ug/L }\end{array}$ \\
\hline 0 & 5.3 & 107 & $<0.05$ \\
\hline 30 & 1.6 & 225 & $<0.05$ \\
\hline 45 & 4.4 & 354 & 0.16 \\
\hline 60 & 5.5 & 370 & 0.07 \\
\hline 90 & 6.7 & 325 & $<0.05$ \\
\hline 120 & 5.9 & 224 & $<0.05$ \\
\hline Normal & $\begin{array}{l}\text { Adequate } \\
\text { stimulation } \\
\text { response }\end{array}$ & $\begin{array}{l}\text { rise by }>170 \\
\text { peak }>450\end{array}$ & $\begin{array}{l}\text { peak }>6.6 \\
3.3-6.6 \\
\text { equivocal }\end{array}$ \\
\hline \hline
\end{tabular}

\section{Management}

Patient was initiated on steroid and growth hormone replacement which was later stopped due to lack of clinical benefit. Testosterone replacement therapy was not indicated given basal gonadotrophin levels. Patient was referred to the fertility clinic for consideration of assisted conception TESE-ICSI (Testicular sperm extraction with intracytoplasmic sperm injection).

\section{Conclusion}

Klinefelter syndrome commonly manifests primarily with hypogonadism and infertility

Hypopituitarism is rarely seen in this condition and only few case reports are described in literature (4). It is unclear if there is a link between chromosomal abnormality and hypopituitarism in Klinefelter syndrome.

Baseline pituitary profile and subsequent dynamic tests should be considered in selected patients.

\section{References}

1. Klinefelter syndrome. (2013). U.S. National Library of Medicine.http://ghr.nlm.nih.gov/condition/ klinefeltersyndrome Retrieved May 20, 2014 2.Bojesen, A, Juul, S., \& Gravholt, C. (2003). Prenatal and postnatal prevalence of Klinefelter syndrome: a national registry study. J Clin Endocrinol Metab, 88, 622-626.

3. Jeannie Visootsak John M Graham Klinefelter syndrome and other sex chromosomal aneuploidies. Orphanet J Rare Dis. 2006; 1: 42. 4.Huges TA ,Borsey DQ Partial hypopituitarism in Klinefelter's syndrome. Br J Clin Pract. 1995 MayJun;49(3):162-3.

Further information :shoibur.rehman@nnuh.nhs.uk 